\title{
RESEARCH
}

\section{SLEEP QUALITY IN PATIENTS OVER 65 YEARS OF AGE IN THE COVID-19 PANDEMIC}

Turkish Journal of Geriatrics

DOI: $10.31086 /$ tigeri.2021.235

2021; 24(3): 381-390

- Ayşe KARAOĞULLARINDAN ${ }^{1}$

- Sanem Okşan ERKAN ${ }^{1}$

- Birgül TUHANIOĞLU1

- Gökhan KURAN ${ }^{1}$

- Orhan GÖRGÜLÜ1

CORRESPONDANCE

${ }^{1}$ Ayşe KARAOĞULLARINDAN

Adana City Training and Research Hospital,

Otolaryngology Head and Neck Surgery

Clinic, Adana, Turkey

Phone: +905068449331

e-mail: draysekara01@gmail.com

Received: May 27, 2021

Accepted: Aug 22, 2021

1 Adana City Training and Research Hospital, Otolaryngology Head and Neck Surgery Clinic, Adana, Turkey

\section{Abstract}

Introduction: While Covid-19 affects people of all ages, elderly patients are at the highest risk. The Covid-19 pandemic has caused sleep disorders and mental health problems as well as physical symptoms.

Materials and Methods: A total of 142 patients, 71 Covid-19 patients hospitalized in the quarantine service and 71 non-covid, were included in the study. Patients were divided into groups of hospitalized in the COVID-19 and non-COVID-19 wards and by age (under 65 and over). The Pittsburg Sleep Quality Index was used to assess sleep quality, the Beck Depression Index to measure the level of depressive symptoms, and the Beck Anxiety Index to measure the level of anxiety symptoms.

Results: The sleep quality of those hospitalized in the COVID-19 ward was worse, and their anxiety and depression symptom scores were higher $(p=$ $0.003, p=0.011$, and $p=0.018$ ). The sleep quality of patients over 65 years of age was significantly worse than that of patients under 65 years of age ( $p$ $<0.001$ ), and anxiety and depression symptom scores of patients over 65 years of age were significantly higher than those of patients under 65 years of age $(p<0.01, p<0.01)$

Conclusion: During the COVID-19 pandemic, anxiety and depression symptoms were observed and sleep quality was deteriorated in patients aged $>65$ years who were placed in quarantine. Hence, this group of patients should be closely monitored in terms of psychological disorders and sleep disorders. If necessary, psychological support and medical treatment should be provided.

Keywords: Covid-19; Pandemic; Anxiety; Depression; Sleep; Geriatrics. 


\section{INTRODUCTION}

Toward the end of 2019, a new coronavirus disease (COVID-19) pneumonia emerged in Wuhan, China, and spread rapidly all over the world. It was eventually announced a pandemic by the World Health Organization (WHO). COVID-19 causes physical and psychological problems due to the high risk of transmission and mortality, of the virus. While COVID-19 affects people of all ages, elderly patients are at the highest risk. Due to the COVID-19 pandemic, the mortality rate of those aged 80 years and above has increased five-fold compared to the global average (1). Therefore, health strategies, such as social distancing, are especially important for preventing the spread of the coronavirus $(2,3)$.

In many countries, patients infected with COVID-19 are treated in isolation wards under strict quarantine conditions. Due to social isolation, uncertainty, physical symptoms, side effects of drugs, fear of infecting others, death of a family member, and negative news on social media, patients with COVID-19 may experience loneliness, anger, anxiety, depression, insomnia, and post-traumatic stress disorder (PTSD) (4). In addition, SARS-CoV-2 can directly reach the brain, disrupt brain functions, and cause mental-psychological problems in patients (5). A recent study found that $96.2 \%$ of clinically stable COVID-19 patients experienced significant PTSD (6). Previous studies that followed patients infected with severe acute respiratory syndrome (SARS) in 2003 showed that the prevalence of PTSD among SARS survivors was $9.79 \%$ in the early recovery phase and $46.2 \%$ at 3 months after discharge, $38.8 \%$ at 12 months (7). Thus, post-pandemic mental distress may be persistent, even after the pandemic is over.

Many studies have reported that patients have negative changes in their sleep patterns and sleep quality due to quarantine $(8,9)$. Few studies have assessed depressive symptoms and impaired sleep quality, especially in elderly patients, during quarantine $(10,11)$. Some standardized questionnaires are used to evaluate anxiety, depression, and sleep quality of patients. The most commonly used among these are the Beck Depression Index (BDI), Beck Anxiety Index (BAI), and Pittsburg Sleep Quality Index (PSQl) (9).

In our study, we aimed to examine to what extent the hospital quarantine applied during the Covid-19 pandemic affected the anxiety and depression symptoms and sleep quality of the patients and whether this effect changes with age.

\section{MATERIALS AND METHODS}

This study was conducted on 71 patients diagnosed with COVID-19 and hospitalized in an isolated covid ward under quarantine conditions and 71 nonCOVID-19 patients hospitalized in non-COVID-19 services, not under quarantine, in the Adana City Training and Research Hospital between February 15, 2021, and March 15, 2021. A total of 142 patients were enrolled in this study. Our study was approved by the ethics committee on January 13, 2021 (number 1256). Written consent was obtained from all patients.

Patients were divided into two groups, i.e., hospitalized in the COVID-19 and non-COVID-19 services, and evaluated them separately according to age (under and above 65 years of age). Patients in the COVID service consisted of stable patients with PCR (+) results, no respiratory distress, and under medical treatment. Patients hospitalized in non-COVID-19 services included stable patients who were deemed suitable for medical treatment. Sleep quality was evaluated using the PSOI, anxiety symptoms using the BAl, and depression symptoms using the BDI. Those younger than 18 years of age, those with previous psychiatric illnesses and sleep disorders, and those who could not complete their questionnaires in their entirety were excluded from the study.

The PSQI was used to evaluate the sleep quality of each patient over the last month, and consistent 
and repeatable PSOI scores were used (12). The PSOl, which enables us to evaluate the sleep quality, as well as the amount, presence, and severity of sleep disorders in individuals through 19 questions, was filled out by the same physician using a one-onone interview with the patients. The PSOI consists of seven items evaluating subjective sleep quality, sleep latency, sleep duration, sleep efficiency, sleep disturbance, and use of sleeping pills; the obtained global score ranges from 0 to 21 , with higher values indicating poor sleep quality and high levels of sleep disturbance. A global score of 5 or above indicates that clinically, sleep quality is significantly worse. Its diagnostic sensitivity and specificity are $89.6 \%$ and $86.5 \%$, respectively $(12,13)$. Agargün et al. adapted the PSOI questionnaire for Turkish patients (13).

The BDI was developed to measure the risk of depression, the level of depressive symptoms, and the change in severity in adults (Beck 1961). The Turkish validity and reliability study was conducted by Hisli (1989). It measures the physical, emotional, and cognitive symptoms of depression. This scale includes 21 symptom categories with four options each. The total score obtained from the inventory indicates the following: 0-9, normal; 10-18, mild symptoms; 19-29, moderate symptoms; and 30-63, severe depression symptoms (14).

The BAI is a self-assessment scale developed by Beck et al. (1988) to determine the frequency of anxiety symptoms experienced by individuals. It evaluates the frequency of anxiety symptoms experienced by an individual. It is a self-rating scale, scored between 0 and 3, which consists of 21 items. The total score obtained from the inventory indicate the following: $0-7$, normal state; 8-15, mild anxiety; 16-25, moderate anxiety; and 26-63, severe anxiety symptoms. Ulusoy et al. (1998) have conducted a Turkish validity and reliability study for this scale (15).

Statistical analyses were performed using SPSS 22.0. A chi-square test was used to compare the findings according to sex. An independent sample t-test, chi-square test, Fisher's exact test, and Mann-Whitney $U$ test were used to compare the findings of patients in COVID-19 and non-COVID-19 services and those under 65 years of age. A multiple linear regression analysis was performed to evaluate the relationship between PSOl, anxiety, and depression.

\section{RESULTS}

A total of 142 patients, 71 hospitalized in the COVID-19 ward and 71 hospitalized in the non-COVID-19 ward were included in the study. A total of 88 patients were below 65 years of age, and 54 patients were above 65 . There were 60 female and 82 male patients.

The sex distributions of the patients hospitalized in the COVID-19 and non-COVID-19 services were homogeneous according to the cut-odd of 65 years of age ( $p=0.070, p=0.075, p=0.431)$. There was no significant difference in terms of the mean age between those hospitalized in the COVID-19 ward (60.01 \pm 11.5 (25-91)) and in the non-COVID-19 ward $(60.46 \pm 11.39(28-86))(p=0.815)$. Similarly, sex distribution was homogeneous $(p=1.00)$ in patients hospitalized in the COVID-19 (female [30 (42.3)]) and non-COVID-19 wards (female [30 (42.3)] ) (Table 1) .

In those hospitalized in the COVID-19 service, individuals aged 65 years or older had a higher PSOI score (equal to or over 5), positive Beck anxiety score, and Beck depression symptoms than those aged $<65$ years $(p=0.026, p<0.001, p=$ 0.005 , respectively). No significant relationship was found in the PSQI among those hospitalized in the non-COVID-19 ward and those aged $\geq 65$ and $<65$ years ( $p>0.05)$. In non-COVID-19 ward inpatients aged $\geq 65$ years, the proportion of those with positive Beck anxiety symptoms and positive Beck depression symptoms was higher than in those aged $<65$ years ( $p<0.001, p<0.001$, respectively). The proportions of those aged $\geq 65$ years, those with 
Table 1: Sex and age distribution of patients hospitalized in Covid and non-Covid services,

\begin{tabular}{|c|c|c|c|c|c|c|c|c|c|c|}
\hline & \multicolumn{3}{|c|}{ Covid services } & \multicolumn{3}{|c|}{ Non-Covid services } & \multicolumn{3}{|c|}{ Total } & \multirow[b]{2}{*}{$P_{\text {group }}$} \\
\hline & $\begin{array}{c}<65 \text { age } \\
(\mathrm{n}: 44)\end{array}$ & $\begin{array}{c}\geq 65 \text { age } \\
(\mathrm{n}: 27)\end{array}$ & Total $(n: 71)$ & $\begin{array}{c}<65 \text { age } \\
(\mathrm{n}: 44)\end{array}$ & $\begin{array}{c}\geq 65 \text { age } \\
(\mathrm{n}: 27)\end{array}$ & Total $(\mathrm{n}: 71)$ & $\begin{array}{c}<65 \text { age } \\
(\mathrm{n}: 88)\end{array}$ & $\begin{array}{c}\geq 65 \text { age } \\
(n: 54)\end{array}$ & Total (n:142) & \\
\hline & $\begin{array}{l}\text { Mean } \pm S D \\
(\min -\max )\end{array}$ & $\begin{array}{l}\text { Mean } \pm S D \\
\text { (min-max) }\end{array}$ & $\begin{array}{l}\text { Mean } \pm \text { SD } \\
(\text { min-max })\end{array}$ & $\begin{array}{l}\text { Mean } \pm S D \\
(\min -\max )\end{array}$ & $\begin{array}{l}\text { Mean } \pm S D \\
\text { (min-max) }\end{array}$ & $\begin{array}{l}\text { Mean } \pm S D \\
(\min -\max )\end{array}$ & $\begin{array}{l}\text { Mean } \pm S D \\
(\min -m a x)\end{array}$ & $\begin{array}{l}\text { Mean } \pm \text { SD } \\
\text { (min-max) }\end{array}$ & $\begin{array}{l}\text { Mean } \pm \text { SD } \\
(\text { min-max) }\end{array}$ & \\
\hline Age & $\begin{array}{c}53.23 \pm 8.14 \\
(25-61)\end{array}$ & $\begin{array}{c}71.07 \pm 6.41 \\
(65-91)\end{array}$ & $\begin{array}{c}60.01 \pm 11.5 \\
(25-91)\end{array}$ & $\begin{array}{c}53.68 \pm 8.13 \\
(28-64)\end{array}$ & $\begin{array}{c}71.52 \pm 5.93 \\
(65-86)\end{array}$ & $\begin{array}{c}60.46 \pm 11.39 \\
(28-86)\end{array}$ & $\begin{array}{c}53.46 \pm 8.09 \\
(25-64)\end{array}$ & $\begin{array}{c}71.30 \pm 6.12 \\
(65-91)\end{array}$ & \begin{tabular}{|c|}
$60.24 \pm 11.40$ \\
$(25-91)$
\end{tabular} & 0.815 \\
\hline Female & $15(34.1)$ & $15(55.6)$ & $30(42.3)$ & $17(38.6)$ & $13(48.1)$ & $30(42.3)$ & $32(36.4)$ & $28(51.9)$ & $60(42.3)$ & \multirow{2}{*}{1.00} \\
\hline Male & $29(65.9)$ & $12(44.4)$ & 41 (57.7) & $27(61.4)$ & 14 (51.9) & 41 (5.7) & $56(63.6)$ & $26(48.1)$ & 82 (57.7) & \\
\hline & \multicolumn{2}{|c|}{$P_{\text {age }}=0.075$} & & \multicolumn{2}{|c|}{$P_{\text {age }}=0.431$} & & \multicolumn{2}{|c|}{$P_{\text {age }}=0.070$} & & \\
\hline
\end{tabular}

Gender: Chi-Square test, Age:Independent Sample t test,

$\mathrm{P}_{\text {age }}$ : Comparison of under 65 and over,

$\mathrm{P}_{\text {group }}$ : Comparison of covid service and non-covid service

PSQI scores $\geq 5$, and those with Beck anxiety and depression symptoms were higher than of those aged $<65$ years $(p=0.018, p<0.001$, and $p<0.001$, respectively). Individuals under 65 years of age admitted in the COVID-19 and non-COVID-19 wards had similar PSOI and anxiety and depression symptoms ( $p>0.05$ ). The rate of PSQI values $\geq 5$ was higher in those aged $\geq 65$ years and those hospitalized in the COVID-19 ward than in those hospitalized in the non-COVID-19 ward $(p=0.011$ and $p=0.028$, respectively). Among individuals aged $\leq 65$ years, the rates of anxiety and depression did not differ between those who were hospitalized in the COVID-19 and non-COVID-19 wards ( $p>0.05$ and $p>$ 0.05 , respectively) (Table 2 ).

Component 7 (daytime functions), PSQI, anxiety, and depression differed between individuals under 65 and over 65 years of age in the COVID-19 ward $(p<0.05)$. The sleep quality of patients over 65 years of age hospitalized in the COVID-19 ward was significantly worse than that of patients under 65 years of age $(p=002)$. Anxiety and depression symptom scores of patients over 65 years of age hospitalized in the COVID-19 ward were significantly higher than those of patients under 65 years of age $(p<0.01$ and $p=0.05$ ). Component 2 (sleep latency), Component 3 (sleep time), Component 4 (sleep efficiency), PSQI, anxiety, and depression differed between individuals $<65$ years of age and $\geq 65$ years of age with non-COVID cervicitis ( $p<0.05$ ). The sleep quality of patients over 65 years of age hospitalized in a non-COVID service was significantly worse than that of patients under 65 years of age $(p=006)$. Anxiety and depression symptom scores of patients over 65 years of age hospitalized in the COVID-19 ward were significantly higher than those of patients under 65 years of age $(p<0.01$ and $p<0.01)$.

Among those aged $<65$ and $\geq 65$ years, Component 1 (subjective sleep quality), Component 2 (sleep latency), Component 3 (sleep time), Component 4 (sleep efficiency), Component 5, (sleep disturbance), Component 7 (daytime functions), PSOI, anxiety, and depression were found to differ ( $p<0$ 05). The sleep quality of patients over 65 years of age was significantly worse than that of patients under 65 years of age $(p<0.001)$, and anxiety and depression symptom scores of patients over 65 years of age were significantly higher than those of 
Table 2: Comparison of PSQl, anxiety and depression symptoms of patients under 65 and over 65 years of age in covid service and non-covid service

\begin{tabular}{|c|c|c|c|c|c|c|c|c|c|c|c|c|c|c|c|c|c|c|c|c|c|c|c|c|}
\hline & \multicolumn{6}{|c|}{ Covid services } & \multirow{3}{*}{$\begin{array}{l}\frac{0}{\sigma} \\
\Omega^{\pi}\end{array}$} & \multicolumn{6}{|c|}{ Non- Covid services } & \multirow{3}{*}{ 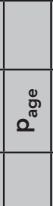 } & \multicolumn{6}{|c|}{ Total } & \multirow{3}{*}{ 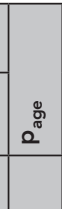 } & \multirow{3}{*}{ 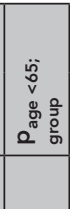 } & \multirow{3}{*}{ 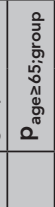 } & \multirow{3}{*}{ 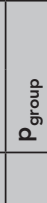 } \\
\hline & \multicolumn{2}{|c|}{$<65$ age } & \multicolumn{2}{|c|}{$\geq 65$ age } & \multicolumn{2}{|c|}{ Total } & & \multicolumn{2}{|c|}{$<65$ age } & \multicolumn{2}{|c|}{$\geq 65$ age } & \multicolumn{2}{|c|}{ Total } & & \multicolumn{2}{|c|}{$<65$ age } & \multicolumn{2}{|c|}{$\geq 65$ age } & \multicolumn{2}{|c|}{ Total } & & & & \\
\hline & $\mathrm{n}$ & $\%$ & $n$ & $\%$ & $n$ & $\%$ & & $n$ & $\%$ & $n$ & $\%$ & $\mathrm{~N}$ & $\%$ & & $n$ & $\%$ & $\mathrm{n}$ & $\%$ & $n$ & $\%$ & & & & \\
\hline \multicolumn{25}{|l|}{ PSOI } \\
\hline$<5$ & 25 & 56.8 & 8 & 29.6 & 33 & 46.5 & \multirow{2}{*}{ స̊. } & 32 & 72.7 & 16 & 59.3 & 48 & 67.6 & \multirow{2}{*}{ 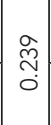 } & 57 & 64.8 & 24 & 44.4 & 81 & 57.0 & \multirow{2}{*}{$\stackrel{\infty}{\circ}$} & \multirow{2}{*}{$\frac{\infty}{\check{\sigma}}$} & \multirow{2}{*}{ 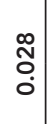 } & \multirow{2}{*}{ бٓ } \\
\hline$\geq 5$ & 19 & 43.2 & 19 & 70.4 & 38 & 53.5 & & 12 & 27.3 & 11 & 40.7 & 23 & 32.4 & & 31 & 35.2 & 30 & 55.6 & 61 & 43.0 & & & & \\
\hline \multicolumn{25}{|l|}{ Beck anxiety Index } \\
\hline anxiety symptoms - & 22 & 50.0 & 2 & 7.4 & 24 & 33.8 & \multirow{2}{*}{$\begin{array}{l}\overline{8} \\
\dot{0} \\
\text { v }\end{array}$} & 29 & 65.9 & 5 & 18.5 & 34 & 47.9 & \multirow{2}{*}{$\begin{array}{l}\overline{8} \\
\dot{0}\end{array}$} & 51 & 58.0 & 7 & 13.0 & 58 & 40.8 & \multirow{2}{*}{$\begin{array}{l}\bar{\delta} \\
\dot{0} \\
\mathrm{v}\end{array}$} & $\bar{m}$ & ㅇ & $\infty$ \\
\hline anxiety symptoms + & 22 & 50.0 & 25 & $9 ., 6$ & 47 & 66.2 & & 15 & 34.1 & 22 & 81.5 & 37 & 52.1 & & 37 & 42.0 & 47 & 87.0 & 84 & 59.2 & & $\overline{0}$ & ठ். & ○. \\
\hline Beck depression Index & & & & & & & & & & & & & & & & & & & & & & & & \\
\hline depression symptom - & 22 & 50.0 & 4 & $1 ., 8$ & 26 & 6,6 & 웅 & 29 & 65.9 & 6 & 22.2 & 35 & 49.3 & $\bar{\delta}$ & 51 & 58.0 & 10 & 18.5 & 61 & 43.0 & $\bar{\delta}$ & $\bar{m}$ & $\stackrel{\sim}{\sim}$ & $\approx$ \\
\hline depression symptom + & 22 & 50.0 & 23 & 8.2 & 45 & 63.4 & 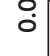 & 15 & 34.1 & 21 & 77.8 & 36 & 50.7 & i & 37 & 42.0 & 44 & 81.5 & 81 & 57.0 & i & б. & $\hat{0}$ & 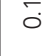 \\
\hline
\end{tabular}

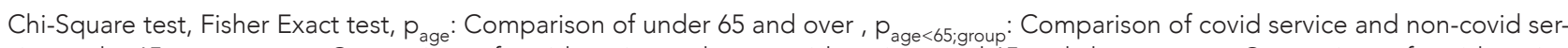
vice under 65, $p_{\text {age } 265 ; \text { group }}$ : Comparison of covid service and non-covid service aged 65 and above, $p_{\text {group: }}$ : Comparison of covid service and non-covid service, PSOI: Pitsburg Sleep Quality Index

patients under 65 years of age $(p<0.01, p<0.01)$. Among those under 65 years old who were hospitalized in the COVID-19 ward and those who were hospitalized in the non-COVID-19 wards, Component 2 (sleep latency), Component 3 (sleep time), Component 5 (sleep disturbance), PSQI, anxiety, and depression differed significantly $(p<0.05)$. Sleep quality was worse in patients under 65 years of age, and anxiety and depression symptoms in the COVID ward scores were higher $(p=0.030, p=$ $0.011, p=0.008$ ). Component 5 (sleep disturbance), Component 7 (daytime functions), and PSOl differed between those hospitalized in the COVID-19 ward and those hospitalized in the non-COVID-19 ward among individuals aged 65 years and older $(p<0.05)$. Sleep quality was worse in those aged 65 years and older who were hospitalized in the COVID-19 ward $(p=0.010)$. When all patients were evaluated, component 2 (sleep latency), Component
3 (sleep time), Component 5 (sleep disturbance), PSQI, anxiety, and depression differed between those hospitalized in the COVID-19 ward and those hospitalized in the non-COVID-19 wards ( $p<0.05$ ). The sleep quality of those hospitalized in the COVID-19 ward was worse, and their anxiety and depression symptom scores were higher $(p=0.003, p=$ 0.011 , and $p=0.018$, respectively) (Table 3 ).

For the PSQI, when sleeping in the COVID-19 ward, age, sex, anxiety, and depression were assessed together, stay in the non-COVID-19 ward decreased the PSOI score. An increase in the depression score in patients aged of 65 years and above also increased the PSQI ( $p<0.05)$. These variables accounted for $30.9 \%$ of the PSQI ( $p<0.001)$.

Increased depression scores were accompanied by increased anxiety scores $(p<0.05)$. This variable accounted for $55.8 \%$ of the anxiety scores $(p<0.001)$. 
Table 3: Comparison of PSQI components, total PSQI scores, anxiety and depression scores of patients under the age of 65 and over from Covid service and non-covid service

\begin{tabular}{|c|c|c|c|c|c|c|c|c|c|c|c|c|c|c|c|c|c|c|c|c|c|c|c|c|}
\hline & \multicolumn{6}{|c|}{ Covid services } & & \multicolumn{6}{|c|}{ Non- Covid services } & \multicolumn{7}{|c|}{ Total } & & & & \\
\hline & \multicolumn{2}{|c|}{$<65$ age } & \multicolumn{2}{|c|}{$\geq 65$ age } & \multicolumn{2}{|c|}{ Total } & \multirow{2}{*}{ 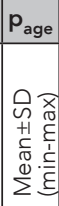 } & \multicolumn{2}{|c|}{$<65$ age } & \multicolumn{2}{|c|}{$\geq 65$ age } & \multicolumn{2}{|c|}{ Total } & \multirow{2}{*}{ 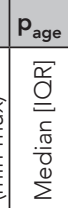 } & \multicolumn{2}{|c|}{$<65$ age } & \multicolumn{2}{|c|}{$\geq 65$ age } & \multicolumn{2}{|c|}{ Total } & & & & \\
\hline & 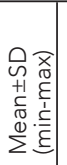 & 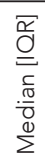 & 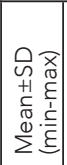 & 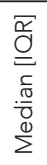 &  &  & & 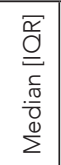 & 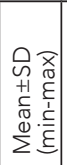 & 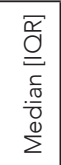 & 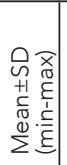 &  & 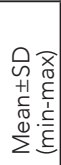 & & 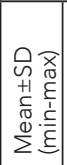 & 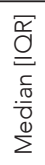 & 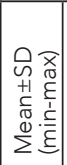 & 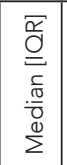 & 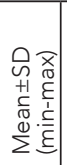 & 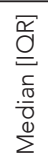 & : & \begin{tabular}{r|r} 
& 0 \\
0 \\
0 \\
0 \\
0 \\
0 \\
0 \\
0 \\
0 \\
0 \\
$e^{0}$
\end{tabular} & 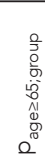 & $\stackrel{\varrho}{\frac{0}{\partial}}$ \\
\hline $\begin{array}{l}\text { Component 1: } \\
\text { subjective sleep } \\
\text { quality }\end{array}$ & $\begin{array}{l}0 \\
0 \\
o \\
+1 \\
\infty \\
\circ \\
0 \\
0\end{array}$ & $\begin{array}{l}\bar{\sigma} \\
\stackrel{\sigma}{\sigma}\end{array}$ & 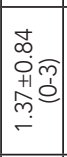 &  & 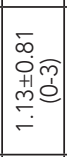 & $\stackrel{\underset{N}{I}}{\check{E}}$ & $\begin{array}{l}\overline{8} \\
0 \\
0\end{array}$ & 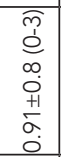 & $\begin{array}{l}\bar{\sigma} \\
\stackrel{c}{\sigma}\end{array}$ & 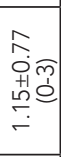 & $\begin{array}{l}\stackrel{\Gamma}{\perp} \\
\stackrel{5}{\leftarrow}\end{array}$ & $\begin{array}{l}\text { ma } \\
\vdots \\
o \\
a \\
o \\
+1 \\
+1\end{array}$ & $\underset{\bar{c}}{\bar{c}}$ & \begin{tabular}{l}
$\infty$ \\
\hdashline \\
\hdashline \\
0
\end{tabular} & 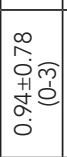 & $\underset{\bar{c}}{\stackrel{\bar{c}}{\sigma}}$ & 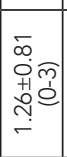 & $\begin{array}{l}\underset{T}{ \pm} \\
\stackrel{\leftarrow}{\leftarrow}\end{array}$ & \begin{tabular}{|c|}
$\tilde{r}$ \\
0 \\
0 \\
$\infty$ \\
0 \\
+1 \\
0 \\
0 \\
-
\end{tabular} & $\stackrel{\underset{T}{\stackrel{I}{\leftrightarrows}}}{\leftarrow}$ & స̦ & 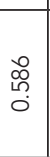 & $\stackrel{L}{0}$ & $\stackrel{\substack{m \\
0}}{o}$ \\
\hline $\begin{array}{l}\text { Component } 2 . \\
\text { Sleep latency }\end{array}$ & 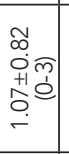 & $\begin{array}{l}\underset{\sim}{N} \\
\stackrel{\text { N }}{0} \\
\stackrel{0}{\sigma}\end{array}$ & $\mid \begin{array}{l}0 \\
\infty \\
0 \\
0 \\
+1 \\
+1 \\
\dot{\sigma} \\
-\end{array}$ & $\stackrel{\underset{N}{\check{L}}}{\check{\sigma}}$ & 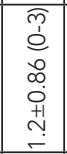 & $\stackrel{\underset{N}{I}}{\check{E}}$ & $\begin{array}{l}\stackrel{\circ}{c} \\
\end{array}$ & $\begin{array}{l}\bar{r} \\
\hat{0} \\
+1 \\
+1 \\
0 \\
0 \\
0 \\
0\end{array}$ & $\begin{array}{l}\overline{\bar{c}} \\
\stackrel{\underline{e}}{\sigma}\end{array}$ & 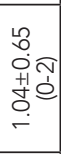 & 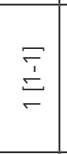 & 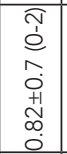 & $\underset{\bar{c}}{\stackrel{\bar{c}}{\sigma}}$ & N్ల & $\mid$\begin{tabular}{l|}
$a$ \\
$\hat{0}$ \\
0 \\
+1 \\
$\infty$ \\
$\infty$ \\
$\infty$ \\
0 \\
0
\end{tabular} & $\underset{c}{\stackrel{F}{c}}$ & 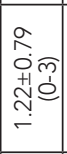 & $\stackrel{\underset{T}{\check{L}}}{\check{\leftarrow}}$ & $\begin{array}{l}\bar{p} \\
0 \\
\infty \\
0 \\
0 \\
+1 \\
0 \\
\dot{+1}\end{array}$ & $\begin{array}{l}\underset{T}{\sigma} \\
\stackrel{o}{-}\end{array}$ & $\stackrel{N}{\circ}$ & $\begin{array}{l}\stackrel{n}{N} \\
0 \\
0\end{array}$ & $\stackrel{\infty}{\circ}$ & $\stackrel{\infty}{\circ}$ \\
\hline $\begin{array}{l}\text { Component 3: } \\
\text { sleep time }\end{array}$ & 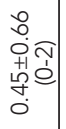 & $\frac{\bar{c}}{\text { ô }}$ & 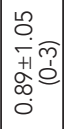 & $\begin{array}{l}\underset{N}{N} \\
\stackrel{0}{-}\end{array}$ &  & ō & $\begin{array}{l}\tilde{\sigma} \\
\delta \\
0\end{array}$ & $\begin{array}{l}\qquad \\
0 \\
0 \\
+1 \\
\infty \\
0 \\
0\end{array}$ & $\begin{array}{l}\overline{0} \\
\text { 임 } \\
\text { o }\end{array}$ & 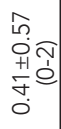 & $\frac{\bar{c}}{\circ}$ & 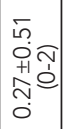 & $\begin{array}{l}\overline{0} \\
\text { 응 } \\
\text { ㅇ }\end{array}$ & \begin{tabular}{l}
$\infty$ \\
\multirow{0}{0}{} \\
0
\end{tabular} & $\begin{array}{l}\infty \\
\sim \\
0 \\
0 \\
+1 \\
\stackrel{+}{\sim} \\
\tilde{n} \\
0 \\
0\end{array}$ & $\frac{\bar{\sigma}}{0}$ & $\mid \begin{array}{l}0 \\
0 \\
0 \\
+1 \\
1 \\
0 \\
0 \\
0 \\
0\end{array}$ & $\frac{\bar{\sigma}}{\stackrel{c}{0}}$ & 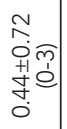 & $\frac{\bar{c}}{0}$ & $\stackrel{\circ}{\circ}$ & : & 응 & : \\
\hline $\begin{array}{l}\text { Component 4: } \\
\text { sleep efficiency }\end{array}$ & $\begin{array}{l}0 \\
0 \\
\circ \\
+1 \\
+1 \\
\stackrel{1}{\sigma} \\
0\end{array}$ & $\frac{\bar{c}}{0}$ & 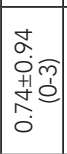 & $\underset{\sigma}{\bar{c}}$ & $\mid \begin{array}{l}0 \\
0 \\
0 \\
+1 \\
+1 \\
0 \\
0 \\
0 \\
0\end{array}$ & $\frac{\bar{\sigma}}{0}$ & $\stackrel{\tilde{N}}{\tilde{\sigma}}$ & 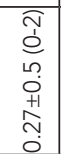 & 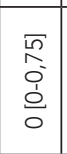 & $\begin{array}{l}a \\
0 \\
0 \\
+1 \\
\text { ᄁo } \\
0 \\
0\end{array}$ & $\begin{array}{l}\bar{c} \\
\text { co }\end{array}$ & $\begin{array}{l}\text { o } \\
0 \\
0 \\
0 \\
0 \\
+1 \\
+ \\
0\end{array}$ & $\frac{\bar{\partial}}{\circ}$ & $\frac{1}{0}$ & $\mid \begin{array}{c}0 \\
\tilde{n} \\
0 \\
+1 \\
+1 \\
0 \\
0 \\
0 \\
0 \\
0\end{array}$ & $\frac{\bar{c}}{0}$ & $\mid \begin{array}{c}\mathfrak{c} \\
0 \\
0 \\
+ \\
+1 \\
01 \\
0 \\
0 \\
0\end{array}$ & $\underset{\leftarrow}{\bar{c}}$ & 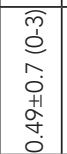 & $\frac{\bar{c}}{0}$ & $\frac{\bar{\sigma}}{0}$ & $\stackrel{\circ}{\circ}$ & $\begin{array}{l}\widetilde{N} \\
\sigma \\
\sigma\end{array}$ & 离 \\
\hline $\begin{array}{l}\text { Component } 5 \\
\text {.Sleep distur- } \\
\text { bance }\end{array}$ & 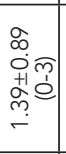 & $\stackrel{\underset{T}{\perp}}{\stackrel{N}{N}}$ & 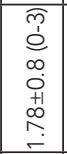 & 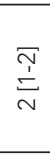 & 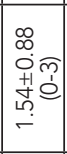 & 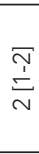 & $\frac{8}{\circ}$ &  &  & 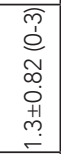 & 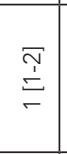 & 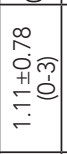 & $\begin{array}{l}\stackrel{\Gamma}{I} \\
\stackrel{\Xi}{\leftarrow}\end{array}$ & $\frac{\grave{0}}{\circ}$ &  & $\begin{array}{l}\underset{T}{\check{I}} \\
\stackrel{\leftarrow}{\leftarrow}\end{array}$ & 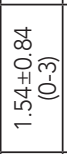 & $\begin{array}{l}\underset{T}{ \pm} \\
\underset{\sigma}{\Xi}\end{array}$ & 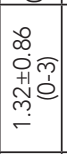 & $\stackrel{\underset{T}{\check{I}}}{\check{\leftarrow}}$ & 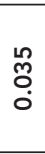 & : & 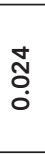 & ัั \\
\hline $\begin{array}{l}\text { Component 6: } \\
\text { drug use }\end{array}$ & 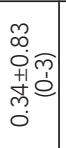 & $\begin{array}{l}\text { 웅 } \\
\text { 응 }\end{array}$ & 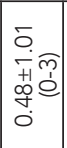 & $\begin{array}{l}\text { o } \\
\text { o } \\
\text { O }\end{array}$ & $\begin{array}{l}\tilde{m} \\
\dot{e} \\
o \\
o \\
0 \\
+1 \\
0 \\
0 \\
0 \\
0\end{array}$ & $\begin{array}{l}\text { o } \\
\text { 응 } \\
\text { O }\end{array}$ & $\begin{array}{l}m \\
\ddot{b} \\
0\end{array}$ & 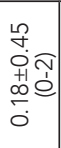 & $\begin{array}{l}\text { 이 } \\
\text { 응 } \\
\text { O }\end{array}$ & 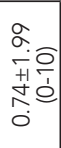 & $\frac{\bar{c}}{\circ}$ &  & $\begin{array}{l}\overline{0} \\
\text { 응 } \\
\text { ㅇ }\end{array}$ & $\stackrel{\stackrel{\infty}{m}}{\circ}$ & $\mid \begin{array}{l}0 \\
0 \\
0 \\
+1 \\
0 \\
0 \\
0 \\
0 \\
0\end{array}$ & $\begin{array}{l}\text { ㅇ } \\
\text { 응 } \\
\text { O }\end{array}$ & $\mid$\begin{tabular}{c}
$n$ \\
\hdashline \\
+1 \\
+1 \\
$\dot{+1}$ \\
0 \\
0
\end{tabular} & $\frac{\bar{\sigma}}{0}$ & 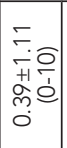 & $\begin{array}{l}\text { 웅 } \\
\text { 응 }\end{array}$ & $\stackrel{\circ}{\circ}$ & $\begin{array}{l}0 \\
0 \\
0 \\
0\end{array}$ & $\begin{array}{l}\hat{m} \\
\text { Oे } \\
0\end{array}$ & $\begin{array}{l}\infty \\
œ \\
\stackrel{0}{0}\end{array}$ \\
\hline $\begin{array}{l}\text { Component } \\
7 \text { : daytime } \\
\text { functions }\end{array}$ &  & $\frac{\bar{\sigma}}{0}$ &  & $\begin{array}{l}\text { F } \\
\text { O } \\
\text { - }\end{array}$ & 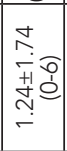 & $\begin{array}{l}\underset{\gamma}{\sigma} \\
\frac{1}{0} \\
0\end{array}$ & $\overline{0}$ & 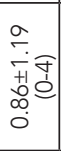 & $\begin{array}{l}\underset{1}{\sigma} \\
\stackrel{0}{0}\end{array}$ & 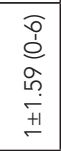 & $\underset{\bar{c}}{\stackrel{F}{c}}$ &  & $\frac{\bar{c}}{\circ}$ & $\begin{array}{l}m \\
\substack{0 \\
0}\end{array}$ & $\mid \begin{array}{l}m \\
m \\
\sim \\
+\neq \\
+1 \\
\infty \\
\infty \\
0 \\
0\end{array}$ & $\frac{\bar{\sigma}}{0}$ & 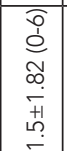 & $\begin{array}{l}\bar{v} \\
\text { d } \\
\sigma\end{array}$ & 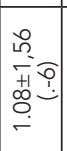 & 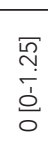 & ¿্口 & 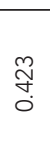 & 弚 & స్ \\
\hline PSOI & 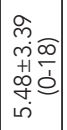 & $\begin{array}{l}\stackrel{n}{\stackrel{n}{n}} \\
\stackrel{m}{n} \\
\omega\end{array}$ & $\mid \begin{array}{l}\stackrel{\sim}{\sim} \\
\dot{+} \\
+\underset{\sim}{+\infty} \\
\underset{+}{+}= \\
\infty \\
\infty\end{array}$ & $\frac{\bar{\sigma}}{\frac{1}{2}}$ & $\begin{array}{l}\infty \\
\alpha \\
\dot{m} \infty \\
+1 \\
\dot{+1} \\
\dot{\sigma} \\
\dot{0}\end{array}$ & $\begin{array}{l}\frac{\sigma}{j} \\
\text { d } \\
0\end{array}$ & $\begin{array}{l}\text { Oे } \\
0 \\
0\end{array}$ & 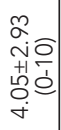 & $\begin{array}{l}\bar{o} \\
\stackrel{2}{N} \\
\stackrel{5}{m}\end{array}$ & 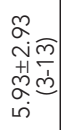 & 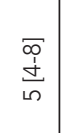 &  & $\begin{array}{l}\underset{\sigma}{0} \\
\dot{m} \\
\dot{\sigma}\end{array}$ & : & 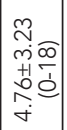 & 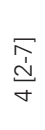 & 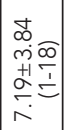 & $\begin{array}{l}\frac{\sigma}{\dot{d}} \\
\frac{\dot{d}}{0}\end{array}$ & 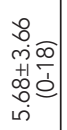 & $\begin{array}{l}\bar{\infty} \\
\dot{m} \\
\dot{m}\end{array}$ & $\begin{array}{l}\overline{8} \\
\dot{0}\end{array}$ & 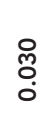 & $\stackrel{\circ}{\circ}$ & ஜ̊ \\
\hline Anxiety &  & $\frac{\sqrt{n}}{\stackrel{\omega}{n}}$ & 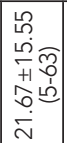 & 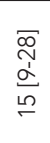 & \begin{tabular}{|l|}
$\infty$ \\
0 \\
$\dot{m}$ \\
+1 \\
+1 \\
$\tilde{m}$ \\
$\dot{m}$ \\
$\stackrel{\rho}{\sim}$ \\
\end{tabular} & $\begin{array}{l}\sigma \\
\frac{\sigma}{\sigma} \\
\dot{b} \\
\sigma\end{array}$ & $\begin{array}{l}\overline{8} \\
\dot{0} \\
\mathrm{v}\end{array}$ & 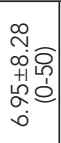 & $\begin{array}{l}\bar{a} \\
\stackrel{i}{\mathfrak{n}} \\
\text { n }\end{array}$ & 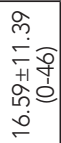 & 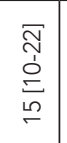 & 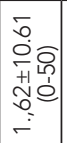 & $\begin{array}{l}\frac{n}{\vdots} \\
\stackrel{\sim}{a} \\
a\end{array}$ & $\begin{array}{l}\overline{8} \\
\dot{0}\end{array}$ & 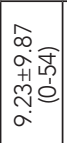 & $\frac{\bar{F}}{\stackrel{m}{0}}$ & 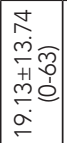 & 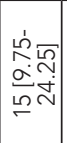 & 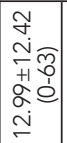 & $\frac{\sqrt[D]{N}}{\stackrel{N}{\bar{n}}}$ & $\begin{array}{l}\overline{8} \\
\dot{0} \\
\mathrm{v}\end{array}$ & $\frac{\bar{\sigma}}{0}$ & $\underset{\hat{n}}{\tilde{O}}$ & $\bar{o}$ \\
\hline Depression & 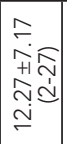 & 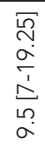 & 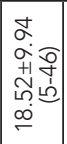 & 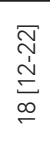 &  & $\begin{array}{l}\underset{\mathbb{N}}{\infty} \\
\stackrel{\alpha}{N} \\
\stackrel{0}{\sim}\end{array}$ & $\stackrel{2}{\circ}$ & 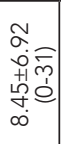 & 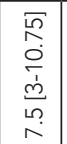 & 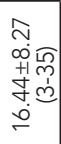 & 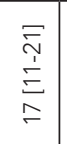 & 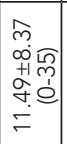 & 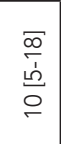 & $\begin{array}{l}\overline{8} \\
\dot{0} \\
\mathrm{v}\end{array}$ & $\mid \begin{array}{l}\hat{N} \\
\hat{+} \\
+1 \\
0 \\
m \\
0 \\
0 \\
0\end{array}$ & $\begin{array}{l}\frac{n}{n} \\
\hat{n} \\
\stackrel{\dot{n}}{\omega} \\
\infty\end{array}$ & 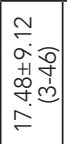 & 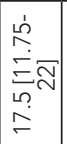 & \begin{tabular}{l|}
$\bar{T}$ \\
$\infty$ \\
+1 \\
+1 \\
0 \\
0 \\
$\dot{m}$ \\
\\
\end{tabular} & 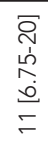 & $\begin{array}{l}\bar{o} \\
\dot{0} \\
\mathrm{v}\end{array}$ & 号 & 贵 & ס \\
\hline
\end{tabular}

Mann Whitney $U$ test, $\mathrm{P}_{\text {age }}$ :comparison of under 65 and over,


aged 65 and above, $p_{\text {group }}$ : Comparison of covid service and non-covid service PSQI: Pitsburg Sleep Quality Index. 
Increased PSQI and anxiety scores also increased the depression score $(p<0.05)$. These variables accounted for $56.9 \%$ of the depression scores $(p<0.001)$ (Table 4).

\section{DISCUSSION}

While medical complications are generally noted during the COVID-19 epidemic, the effects of SARSCoV-2 on mental health and sleep quality should not be ignored. Considering that many psychiatric disorders were noted after the SARS-CoV-1 epidemic in 2002-2003, the effects of the SARS-CoV-2 epi- demic on the mental health should be examined. In our study, we found that patients over 65 years of age generally had worse sleep quality and higher anxiety and depression symptom scores. Being in the COVID-19 ward directly affected sleep quality negatively, especially in patients over the age of 65 years, and caused sleep disturbances. In addition, we found that the anxiety and depression symptoms of the patients in the COVID-19 ward were significantly higher than of those in the non-COVID-19 ward, especially in those aged $>65$ years.

In a meta-analysis on the effects of the COV-

Table 4: The relationship between PSQI, anxiety and depression scores, age, gender, service and each other

\begin{tabular}{|c|c|c|c|c|c|c|}
\hline & & \multicolumn{2}{|c|}{$\begin{array}{c}\text { Unstandardized } \\
\text { Coefficients }\end{array}$} & \multirow{2}{*}{$\begin{array}{c}\begin{array}{c}\text { Standardized } \\
\text { Coefficients }\end{array} \\
\text { Beta } \\
\end{array}$} & \multirow{2}{*}{$t$} & \multirow{2}{*}{$p$} \\
\hline & & B & Std. Error & & & \\
\hline \multirow{6}{*}{$\begin{array}{l}\text { PSOI } \\
\mathrm{R}^{2}: 0,309 \\
\mathrm{P}<0,001\end{array}$} & (Constant) & 3.903 & 0.603 & & 6.472 & $<0.001$ \\
\hline & Non-covid services & -1.237 & 0.533 & -0.170 & -2.321 & 0.022 \\
\hline & Age $(\geq 65)$ & 1.188 & 0.596 & 0.158 & 1.993 & 0.048 \\
\hline & Sex (female) & -0.644 & 0.539 & -0.087 & -1.194 & 0.235 \\
\hline & Anxiety & 0.045 & 0.031 & 0.154 & 1.444 & 0.151 \\
\hline & Depression & 0.125 & 0.044 & 0.297 & 2.815 & 0.006 \\
\hline \multirow{6}{*}{$\begin{array}{l}\text { Anxiety } \\
\mathrm{R}^{2}: 0,558 \\
\mathrm{p}<0,001\end{array}$} & (Constant) & -1.806 & 1.868 & & -0.967 & 0.335 \\
\hline & Non-covid services & -1.363 & 1.472 & -0.055 & -0.926 & 0.356 \\
\hline & Age $(\geq 65)$ & 2.409 & 1.630 & 0.094 & 1.478 & 0.142 \\
\hline & Sex (female) & 2.842 & 1.453 & 0.113 & 1.956 & 0.053 \\
\hline & Depression & 0.877 & 0.098 & 0.615 & 8.908 & $<0.001$ \\
\hline & PSQI & 0,334 & 0.231 & 0.098 & 1.444 & 0.151 \\
\hline \multirow{6}{*}{$\begin{array}{l}\text { Depression } \\
R^{2}: 0,569 \\
p<0,001\end{array}$} & (Constant) & 4.707 & 1.233 & & 3.819 & $<0.001$ \\
\hline & Non-covid services & -0.347 & 1.021 & -0.020 & -0.340 & 0.734 \\
\hline & Age $(\geq 65)$ & 1.952 & 1.124 & 0.109 & 1.736 & 0.085 \\
\hline & Sex (female) & -0.402 & 1.019 & -0.023 & -0.394 & 0.694 \\
\hline & PSQI & 0.441 & 0.157 & 0.185 & 2.815 & 0.006 \\
\hline & Anxiety & 0.420 & 0.047 & 0.599 & 8.908 & $<0.001$ \\
\hline
\end{tabular}

p:Multiple Linear Regression

PSQI: Pitsburg Sleep Quality Index, 
ID-19 pandemic on mental health, 43 studies were examined, and only two of them evaluated patients infected with COVID -19. Of these 43 studies, 41 were conducted on healthcare workers and the general population. In the two studies on COVID-19 patients, high levels of post-traumatic stress symptoms (PTSS) (96.2\%) and significantly higher depressive symptoms ( $p=0.016$ ) were observed (16).

In a study by Zhang et al. in COVID-19 patients, $20.9 \%$ and $18.6 \%$ had symptoms of anxiety and depression, respectively. In Guo et al.'s study, 38.8\%, $45.9 \%$, and $54.1 \%$ of patients had symptoms of anxiety, depression, and a sleep disorder, respectively. Finally, Dai et al. found symptoms of anxiety and depression and poor sleep quality in $18.6 \%, 13.4 \%$, and $84.7 \%$ of patients, respectively $(17,8,9)$. Female sex, having colleagues infected with COVID-19, poor sleep quality, more than two physical symptoms of anxiety, having a family member infected with COVID-19, and more than two physical symptoms of Covid-19 were identified as the greatest risk factors for depression $(9,17)$. In addition, the quarantine period and some inflammatory markers may affect these parameters (17). In our study, 66.2\%, $63.4 \%$, and $53.3 \%$ of patients in the COVID-19 ward had symptoms of anxiety, depression, and sleep disturbances, respectively. Thus, the results of these studies on anxiety and depression and the factors affecting them are discrepant. In our study, being in quarantine and being over 65 years of age increased the symptoms of anxiety and depression and negatively affected the sleep quality. Our study was conducted in patients hospitalized in isolated COVID-19 wards. Patients were kept in quarantine in single rooms for an average of 10-14 days until their treatment was completed, and nobody could enter or leave the room except for the medical staff. In addition, communication was facilitated through room phones. There was only one companion allowed, especially for elderly patients who could not perform self-care. The patients remained secluded from their relatives, friends, and social circles dur- ing their hospitalization periods. It is impossible for social isolation not to adversely affect the mental health of patients (18). Many studies have reported that curfew, quarantine, and isolation conditions increase the symptoms of anxiety and depression (18).

A previous study reported that COVID-19 patients hospitalized with mild symptoms showed higher levels of depression, anxiety, and PTSS than non-COVID-19 controls (8). In our study, patients hospitalized in the COVID-19 ward showed a significantly higher rate of anxiety and depression symptoms than those hospitalized in non-COVID-19 wards; in addition, their sleep quality was worse. In our study, we did not assess PTSS but evaluated the sleep quality of the patients according to seven components of the PSQI and the total PSQI score. We found that the sleep quality of patients hospitalized in the COVID-19 ward, especially those over 65 years of age, was worse. We found that these patients had worse daytime functions. Moreover, they had difficulty staying awake during daily activities such as drinking, eating, and socializing; they also had reluctance in doing these activities regularly.

It was observed that COVID-19 patients showed higher symptoms of anxiety and depression in the first week of hospitalization, and that these symptoms decreased 1 month following hospitalization (19). Some studies have reported mental health problems and changes in sleep quality, even after hospital discharge (20). Some studies have emphasized the importance of social support after discharge, as well as during hospitalization (20). We followed the patients during hospitalization and did not assess them after discharge. COVID-19 patients should be followed up even after discharge, and if necessary, the duration of psychological support should be extended.

Bao et all, $49.8 \%$ of patients reported that psycho-education services provided in the hospital were useful (6). Given the detrimental impact of PTSS, appropriate psychological interventions and 
long-term follow-up evaluations for COVID-19 patients should be urgently initiated. Healthcare professionals, particularly clinical nurses, should be aware of the psychological state of COVID-19 patients and promote resilience to improve their mental health. Mental and physical health in the elderly is adversely affected by social isolation. Therefore, a multi-component program that includes exercise and psychological strategies is highly recommended for this population during quarantine. In our study, psychiatric consultation was requested for patients with clinical psychological disturbances, and medical treatment was initiated if necessary. However, these were not recorded during the study and were excluded. Further studies are needed on patients aged $>65$ years who are admitted in the COVID-19 ward and need psychological support.

Ozamis et al. measured anxiety, stress, and depression levels in a sample of 976 adults using the Depression Anxiety and Stress Scale (DASS) during the COVID-19 pandemic. They observed higher levels of anxiety, depression, and stress, especially in people under 60 years of age and in those with chronic conditions (18). They also stated that the anxiety and depression symptoms of the patients increased after the quarantine order was given. The authors recommend psychological support to alleviate the psychological effects of the pandemic. Similarly, we found that quarantine conditions increased the symptoms of anxiety and depression in our study. In our study, unlike the Ozamycin study,

\section{REFERENCES}

1. Bialek S, Boundy E, Bowen V. et all. Severe Outcomes Among Patients with Coronavirus Disease 2019 (COVID-19) — United States, February 12March 16, 2020. MMWR Morb Mortal Wkly Rep. 2020;69(12):343-346. (PMID: 32214079)

2. Rudolph CW, Zacher H. "The COVID-19 Generation": A Cautionary Note. Work Aging Retire. 2020;XX(Xx):1-7. (DOI:10.1093/workar/waaa009)

3. Cudjoe TKM, Kotwal AA. "Social Distancing" Amid a anxiety and depression symptoms were found to be higher in the geriatric population. These differences may be due to the study population, age of the patients, presence of chronic disease, quarantine status, hospitalization, and COVID-19 positivity.

This study had several limitations. We did not assess patients' comorbid diseases and quarantine periods. In addition, we did not record anxiety and depression symptoms or the sleep quality of the patients after discharge. Our study was conducted in a small population, and more extensive studies in larger populations may yield more robust conclusions.

\section{CONCLUSION}

During the COVID-19 pandemic, anxiety and depression symptoms were observed and sleep quality was deteriorated in patients aged $>65$ years who were placed in quarantine. Hence, this group of patients should be closely monitored in terms of psychological disorders and sleep disorders. If necessary, psychological support and medical treatment should be provided.

CONFLICT OF INTEREST STATEMENT: None

ACKNOWLEDGMENT: Thanks to Asena Ayça Özdemir from Mersin University Statistics Department for statistical analysis.

Crisis in Social Isolation and Loneliness. J Am Geriatr Soc. 2020 Jun; 68(6):E27-E29. (PMID: 32359072)

4. Zhou SJ, Zhang LG, Wang LL et all. Prevalence and socio-demographic correlates of psychological health problems in Chinese adolescents during the outbreak of COVID-19. Eur Child Adolesc Psychiatry. 2020 Jun;29(6):749-758. (PMID: 32363492)

5. Holmes EA, O'Connor RC, Perry VH et all. Multidisciplinary research priorities for the COVID-19 pandemic: a call for action for mental health science. Lancet Psychiatry. 2020 Jun;7(6):547-560.(PMID: 


\section{9)}

6. Bo HX, Li W, Yang $Y$ et all. Posttraumatic stress symptoms and attitude toward crisis mental health services among clinically stable patients with COVID-19 in China. Psychol Med. 2020 Mar 27:1-2. (PMID: 32216863)

7. U. Wesemann, N. Hadjamu, G. Willmund S. et all. Influence of COVID-19 on general stress and posttraumatic stress symptoms among hospitalized high-risk patients Psychol Med. 2020 Aug 14 :1-2. (PMID: 32794442)

8. Guo Q, Zheng Y, Shi J et all. Immediate psychological distress in quarantined patients with COVID-19 and its association with peripheral inflammation: A mixed-method study. Brain Behav Immun. 2020 Aug;88:17-27. (PMID: 32416290)

9. Dai LL, Wang $X$, Jiang TC et all. Anxiety and depressive symptoms among COVID-19 patients in Jianghan Fangcang Shelter Hospital in Wuhan, China. PLoS One. 2020 Aug 28;15(8):e0238416. (PMID: 32857826)

10. Sepúlveda-Loyola W, Rodríguez-Sánchez I, PérezRodríguez $\mathrm{P}$ et all. Impact of Social Isolation Due to COVID-19 on Health in Older People: Mental and Physical Effects and Recommendations. J Nutr Health Aging. 2020;24(9):938-947. (PMID: 33155618)

11. Elezi F, Tafani G, Sotiri E, et all. Assessment of anxiety and depression symptoms in the Albanian general population during the outbreak of COVID-19 pandemic. Indian J Psychiatry. 2020 Sep;62(Suppl 3):S470-S475. (PMID: 33227057)

12. Buysse DJ, Reynolds CF 3rd, Monk TH et all. The Pittsburgh Sleep Quality Index: a new instrument for psychiatric practice and research. Psychiatry Res. 1989 May;28(2):193-213. (PMID: 2748771)

13. Agargun MY, Kara $H$, Anlar $O$. The validity and reliability of Pittsburgh Sleep Quality Index. Turkish Psychiatry Rev 1996; 7(Suppl 2): 107-15. (in Turkish) [Internet] Available from: http://www.turkpsikiyatri. com/en/default.aspx? modul=summary\&id $=210$. Accessed:16.04.2020.

14. Wathelet M, Duhem S, Vaiva $G$ et all. Factors Associated With Mental Health Disorders Among University Students in France Confined During the COVID-19 Pandemic. JAMA Netw Open. 2020 Oct 1;3(10):e2025591.( PMID: 33095252)

15. Ulusoy M, Sahin NH, Erkmen H. The Turkish version of the Beck Anxiety Inventory: Psychometric properties. J Cogn Pschother 1998;12:163-72. (In Turkısh). [ Internet ] Avaliable from https://www.researchgate. net/publication/233792003_Turkish_Version_of the_Beck_Anxiety_Inventory_Psychometric_Properties

16. Vindegaard N, Benros ME. COVID-19 pandemic and mental health consequences: Systematic review of the current evidence. Brain Behav Immun. 2020 Oct;89:531-542. (PMID: 32485289)

17. Zhang J, Yang $Z$, Wang $X$ et all. The relationship between resilience, anxiety and depression among patients with mild symptoms of COVID-19 in China: A cross-sectional study. J Clin Nurs. 2020 Nov;29(2122):4020-4029. (PMID: 32702192)

18. Ozamiz-Etxebarria N, Dosil-Santamaria M, Picaza-Gorrochategui $M$ et all. . Stress, anxiety, and depression levels in the initial stage of the COVID-19 outbreak in a population sample in the northern Spain. Cad Saude Publica. 2020 Apr 30;36(4):e00054020. (PMID: 32374806)

19. Matalon N, Dorman-Ilan S, Hasson-Ohayon I et all.. Trajectories of post-traumatic stress symptoms, anxiety, and depression in hospitalized COVID-19 patients: A one-month follow-up. J Psychosom Res. 2021 Feb 16;143:110399. (PMID: 33618149)

20. Liu D, Baumeister RF, Veilleux JC et all. Risk factors associated with mental illness in hospital discharged patients infected with COVID-19 in Wuhan, China. Psychiatry Res. 2020 Oct;292:113297..( PMID: 32707218) 